\title{
Evaluation of genes and molecular pathways involved in the development of cardiovascular disease in preeclampsia patients: biological system and bioinformatics analysis approach
}

Shahrzad Amirlatifi ( $\nabla$ e.amrovani@gmail.com)

Iran University of Medical Sciences: Tehran University of Medical Sciences

Mahboubeh Pazoki

Iran University of Medical Sciences

Mehran Amrovani

2- High Institute for Education and Research in Transfusion Medicine, Tehran, Iran.

Shima ghezelbash

Iran University of Medical Sciences, Tehran, Iran.

Fatemeh Dastyar

School of Medicine Bushehr University of Medical Sciences

\section{Research Article}

Keywords: Preeclampsia, Hypertension, Cardiovascular Disease, System Biology

Posted Date: March 7th, 2022

DOI: https://doi.org/10.21203/rs.3.rs-1408549/v1

License: (c) (1) This work is licensed under a Creative Commons Attribution 4.0 International License.

Read Full License 


\section{Abstract}

Background: Hypertension in preeclampsia (PE) is one of the most important complications in these patients, which increases the risk of cardiovascular disease (CVD). Bioinformatics analysis can identify the signaling pathways, genes and microRNAs involved in the development of CVD due to hypertension; it can also reduce the risk of developing PE by providing appropriate target therapies.

Method: In this study, after collecting data and determining the gene list, changes in gene expression in patients with severe and non-severe forms of PE were compared. Protein-Protein interaction (PPI) and pathway enrichment analysis were also used to evaluate the common genes and pathways between the two cited forms of PE.

Result: FGF, TNF, Adherent junction, metabolic signaling pathway, GATA3-mediated activation pathways of Th2, EGF / EGFR signaling pathway and Rab GTPase among the most important signaling pathways are effective in CVD development. HLTF, SUM01, KDR, SNRPD3, DERL2, VCP, EIF4B, NOTCH1, SOCS3, CBL, ICAM-1, ITGB2, STRN, MEF2A and PTPRC are the most important genes and miR-3135-3P, miR-505, miR-6088, miR-451, miR-4637-3P, miR-1281-3P, miR-4304-3P, miR-323-3P, miR-3150-3p.1, miR-4718, miR151-5p, miR-3117-5p.2, miR-3613, miR-1278- 3P, miR-4467, miR-4730 , miR-190b-3p , miR-610-5p, miR3648-3p , miR-4796-3p, miR-2277-3P, miR-325, miR- 6807-3p , miR-551a-3p, and miR-3131-3P have been also effective in the development of CVD by hypertension in PE patients.

Conclusion: Finally, it was concluded that identification of common signaling pathways, genes and microRNAs between severe and non-severe forms of PE can be effective to design preventive and treatment strategies for CVD caused by hypertension.

\section{Introduction}

Preeclampsia (PE) is one of the most common diseases, that occurs in pregnant women during pregnancy. Its prevalence is increasing in developing countries. Based on diagnostic criteria, as well as the study population, about 1.8-16.7 new cases of PE are identified annually in cited countries (1). PE is clinically divided into two types, mild and severe. Because severe cases are associated with more severe clinical symptoms, worse maternal and fetal outcomes, higher risk of developing PE and higher risk of recurrence, it seems that the pathogenesis of mild to severe PE can vary $(2,3)$. Depending on the pathogenesis and the severity of the disease, different symptoms are observed in patients.

It has been shown, that hypertension is one of the main and common symptoms in patients. Based on this finding and the obtained data from recent studies, it has been shown that the incidence of hypertension in severe form is lower compared to patients with mild symptoms. However, it has been shown that the development of hypertension in patients can increase the risk of cardiovascular disease (CVD) (4). Given that, preventing high blood pressure can prevent harm to mother and fetus, so it is effective to identify the pathways, that are involved in diagnosing the pathogenesis of CVD due to hypertension (5). 
System biology is an approach by which the pathways and genes involved in the pathogenesis of the disease can be identified, and patient survival can be increased by applying treatment strategies and pathways. Using this approach can identify diagnostic biomarkers and high-risk individuals(6). So far, very few studies have been performed to identify the pathways and genes involved in the pathogenesis of CVD due to hypertension in patients; so this study, for the first time examined the molecular pathways and genes involved in the development of CVD in PE patients through biological system approach.

\section{Materials And Methods}

\section{Gene Expression Omnibus (GEO) datasets and bioinformatics data preparation}

This study was provided with bioinformatics analysis. We first used the GEO database and selected the appropriate microarray-dataset for this study. GSE48424, GSE99007, and GSE91189 datasets (table 1) consist of 36 samples about the non- severe, severe PE and healthy individuals. The used platforms in this dataset was GPL6480 Agilent-014850 Whole Human Genome Microarray 4x44K G4112F (Probe Name version). After defining the groups using the GEO2R tool, we isolated the differential expression profiles of the genes and saved them in an Excel file. Then we separated the gene clusters with up and down expression, and prepared them for the next step. In this part, the P-value $₫ 0.05$ was considered as the significance level (Fig. 1).

\section{Assessment of signaling pathways and gene ontology}

In this section high and low expressed genes were chosen from dataset isolated. After that, via the Venn diagram, the common genes profiles between non-severe and severe PE were determined. In the next step, the obtained common genes were uploaded to the Enrichr database to examine the signaling pathways and gene ontology. The KEGG and bioplanet libraries were used to analyze the signaling pathways. The ontology section was then used to evaluated the molecular functions, and biological processes of high and low-expressed genes. The Shiny GO database was also used to plot the communication network between the results. In this part, the P-value $₫ 0.05$ was considered as the significance level.

\section{Evaluation of protein- protein interactions (PPI)}

After evaluating the signaling pathways and gene ontology, the effective pathways in non-severe to severe PE were selected. The relationship between their protein networks was assessed using the STRING database.

\section{Candidate miRNAs selection}

At this point, after isolating important proteins in the PE-dependent signaling pathways, we used the MienTurnet database to examine the miRNAs associated with these genes. In this part, the P-value $\mathbb{0} 0.05$ was also considered as statistically significant. 


\section{Results}

\section{Evaluation of most prominent signaling pathways between non-severe and severe preeclampsia}

After clustering the genes for dataset, 235 high and 546 low-expressed common genes were obtained in non-severe and severe form of PE. Activation of calcium-permeable kainate receptor, steroid biosynthesis, cytochrome P450 metabolism of vitamins, GATA3-mediated activation of Th2 cytokine expression, SLC26 family multifunctional anion exchangers, FGFR function and FGFR3b ligand binding and activation signaling pathways were observed in high-expressed genes. Rab GTPases mark targets in the Endocytosis machinery, COPII-mediated vesicle transport, PIP biosynthesis at the late endosome membrane, gene expression regulation by hypoxia-inducible factor, JNK (c-Jun kinases) phosphorylation and activation mediated by activated human TAK, EGF/EGFR signaling pathway, TNF signaling and adherent junctions signaling were observed in low- expressed genes (Fig. 2).

\section{Evaluation of gene ontology between non- severe and severe preeclampsia}

This section further evaluates the common genes from the previous step, and examines their biological processes. Accordingly, mitochondrial translational elongation, mitochondrial translational termination, mitochondrial gene expression and translational elongation were observed in high expressed genes. Also, for low-expressed genes, endoplasmic reticulum to Golgi vesicle-mediated transport, vesicle organization, Golgi vesicle transport, organelle assembly, regulation of organelle organization, cytoskeleton organization, establishment of protein localization, establishment of localization in cell, intracellular signal transduction, cellular protein modification process, and macromolecule modification were associated with biological processes (Fig. 3, table 2 and 3 ).

\section{Association proteins between non- severe, severe preeclampsia with placenta and chorion}

The communication network between the common proteins of high and down expressed genes of nonsevere, severe PE with placenta and chorion was plotted. High-expressed HLTF, SUMO1, KDR, SNRPD3, DERL2, VCP, and EIF4B proteins and downregulated proteins of NOTCH1, MAML1, SOCS3, CBL, ICAM1, ITGB2, STRN, MEF2A, and PTPRC had the most relationship between severe and non-severe PE signaling pathways (Fig. 1 and table 4).

\section{Evaluation of candidate miRNAs between non-severe and severe preeclampsia with placenta and chorion}

In this part of bioinformatics analysis, the strong association of miRNAs with common high and low expressed genes was investigated. In the regulation of genes with elevated expression, hsa-miR-3135- 
3p/hsa-miR-505/hsa-miR-6088, hsa-miR-451, hsa-miR-4637-3p/hsa-miR-1281-3p, hsa-miR-4304-3p/hsamiR-323-3p, hsa-miR-3150-3p.1, hsa-miR-4718/hsa-miR-151-5p, hsa-miR-3117-5p.2, hsa-miR-3613, and hsa-miR-1278-3p. The common miRs that had reduced expression in non-severe and severe PE were hsamiR-4467, hsa-miR-4730, hsa-miR-190b-3p, hsa-miR-610-5p, hsa-miR-505-3p, hsa-miR-3648-3p/hsa-miR4796-3p, hsa-miR-2277-3p, hsa-miR-325/hsa-miR-6807-3p, hsa-miR-551a-3p, and hsa-miR-3131-3p (Figs. 4 and 5).

\section{Discussion}

$\mathrm{PE}$ is one of the most important causes of mortality in pregnant women. Hypertension is also one of the leading causes of death in pregnant women with PE. Hypertension can increase the risk of CVD and subsequent mortality in pregnant women. By identifying the signaling pathways, genes and microRNAs involved in the development of CVD due to hypertension in pregnant women with $\mathrm{PE}$, the risk of developing CVD due to hypertension can be reduced; it is achievable by providing appropriate target therapies, and consequently the mortality rate will be reduced. Therefore, in this study, involved signaling pathways, genes and microRNAs in the development of CVD caused by hypertension in pregnant women with PE were investigated using the biological system.

\section{Signaling Pathways}

The findings of this study indicated that important signaling pathways involved in inducing hypertension and subsequent CVD are FGF, TNF, Adherent junction, metabolic signaling pathway, GATA3-mediated activation of Th2, EGF / EGFR signaling and Rab GTPase pathway.

In the FGF signaling pathway, binding of FGF to the FGFR3 receptor by activating the TEL / FGFR3 / Ras / Raf / MEK1 / ERK / RSK2 pathway can induce proliferation in Arterial Vascular Smooth Muscle Cells (VSMCs). Subsequent induction of proliferation in arterial VSMCs induces angiogenesis, that may play a role in the development of atherosclerosis and CVD following hypertension (7). TNF / TNFR1 / TRADD / FADD / Caspase8,10 / Caspase3 and TNF / TNFR1 / P55 / TRADD / FADD / RIP / NEMO / IKK / NF-K $\beta$ pathways in the downstream of the TNF signaling can induce apoptosis and inflammation in Endothelial Cell (EC).

Following induction of apoptosis and inflammation in EC, EC dysfunction occurs, resulting in CVD due to hypertension (8). Adherent joints play an important role in the connections between ECs. One of the most important adherent junctions in intercellular connections is VE-Cadherin. During the inflammatory process, the IL-1 / sAC / cAmp / CREB / CRE2,6 / VE-Cadherin pathway leads to non-copying of the VECadherin molecule, and subsequently plays an important role in the destruction of adherent junctions. Consequence of loss of adherent junction during inflammation is EC dysfunction and subsequent CVD due to hypertension (9).

In a study by Koga et al., the results showed that an increase in VE-Cadherin levels was associated with EC dysfunction and subsequent Coronary Artery Disease (10). Metabolic pathways are also involved in 
CVD due to hypertension. One of the most important of these metabolic pathways is the steroid synthesis pathway. Subsequently, during uterine hypoxia, steroids through the MEK / ERK1,2 / PKC pathway can affect uterine arterial contraction; thus it could be effective in hypertension during PE (11).

In the GATA3-mediated activation of Th2 signaling pathway, GATA3 is a transcription factor, that plays an important role in differentiating Th0 cells from Th2. As Th2 cells evolve by GATA3 transcription factor, interleukins 4 and 13 are subsequently secreted from Th2 cells. IL4 and IL13 can play a significant role in hypertension and CVD induction. Thus, IL-4 via the IL4R / JAK3 / STAT6 / NOX2 / NOX4 / ROS / AP-1 / Col1a pathway and IL-13 through the IL13Ra / TYK2 / JAK2 / STAT6 signaling pathway in ECs can also induce inflammation, and subsequently EC dysfunction. IL-4 and IL-13 can also induce fibrosis in cardio myocytes through the mentioned signaling pathways. The consequence of fibrosis in cardio myocytes and EC dysfunction is hypertension and CVD induction. in addition, IL-13 can induce hypertension through the Smad2 / 3 / TGF- $\beta$ signaling pathway.

In a study by Shah et al., it was found that GATA3 plays an important role in inducing myocardial infraction (MI) and cardiac hypertrophy; so that, cardiac function have been improved in mice with mutations in GATA3, and the incidence of $\mathrm{Ml}$ and hypertrophy have been reduced $(12,13)$.

Binding of Epidermal Growth Factor (EGF) to its receptor in the EGF / EGFR signaling pathway induces NOX1 / P22phox / NOXa1 / NOX01 / O2- / NF-K $\beta$ signaling, which produces Pro-inflammatory cytokine. These inflammatory cytokines lead to inflammation in the EC, and subsequently can induce hypertension through EC dysfunction. In addition, Angiotensin II can activate the EGFR signaling pathway, and activates the Shr / Grb2, Ras / ERK and PI3K / Akt / P70S6 Kinase pathways through EGFR / ErbBs. Consequence of activation of EGFR signaling pathway and other downstream pathways induced by Angiotensin II signaling pathway is hypertension. In a study by Semplicini et al., the findings showed that by suppressing the Src / EGFR / Akt signaling pathway induced by Angiotensin II, cardiac hypertrophy and subsequent hypertension could be prevented $(14,15)$.

Rab GTPase is an enzyme, that converts the Rab GDP to Rab GTP. There are different types of this enzyme, including Rab1 and Rab7. Studies have shown, that Rab1 and Rab7 in the Rab GTPase signaling pathway can play a significant role in hypertension. Thus, Rab GTPase enzyme of Rab7 type can disrupt arterial EC function through the Ras / Raf / MEK / ERK signaling pathway, and consequently induces hypertension and Aortic Dissection (AD). Rab1, another type of Rab GTPase enzyme may also be involved in the transfer of Angiotensin II type 1 Receptor (AT1R) from the endoplasmic reticulum to the Golgi apparatus, and subsequently to EC levels. In addition, Rab1 enzyme mediates the STAT3 signaling pathway by transporting AT1R to the EC surface, thereby inducing hypertension. Transfer of the AT1R receptor to the EC surface by Rab1 GTPase can also lead to hypertension via the signaling pathway induced by Angiotensin II binding to this receptor.

By Angiotensin II binding to the AT1R receptor at the EC level, the p-Akt / ERK1 / 2 signaling pathway is induced, resulting in inflammation. On the other hand, Rab GTPase enzyme can induce hypertrophy and subsequent hypertension through the PKC / PKA / p-PKD / HDAC and PKC / PKA / PKD-P / AKAP / Lbc / 
p38MAPK / mTOR signaling pathways. A study by Dorn et al. showed that increased expression of RabGTPase in cardio myocytes could be impressive in inducing myocardial hypertrophy and subsequent hypertension (16-18).

\section{Genes}

In addition to signaling pathways, bioinformatics analyzes of this study also examined the genes involved in the development of CVD due to hypertension in PE individuals. According to bioinformatics findings, the most important genes involved in the development of hypertension and subsequent induction of CVD in PE are as following:

HLTF, SUM01, KDR, SNRPD3, DERL2, VCP, EIF4B, NOTCH1, MAML1, SOCS3, CBL, ICAM1, ITGB2, STRN, MEF2A and PTPRC.

The HLTF gene is linked to P53 gene via the POLi / P53complex / HLTF / ZRANB3 pathway.

Subsequently P53 genes in the upstream of the HLTF gene signaling pathway can inhibit cell proliferation by suppressing the cell cycle. P53 can inhibit proliferation in EC via the P53 / miR192 / miR215 / CDC7 / MAD2L1 / CUL5, P53 / miR34a / b / c / CDC4 / 6 / Cyclin E and P53 / miR145 / CDK6 signaling pathways. Subsequently, miR-31a-5p could induce proliferation in EC by suppressing P53 inhibitory signaling. On the other hand, P53 can induce apoptosis in the EC in the downstream of the Alm / pSer15p53 / Puma / Bax / Bak / Caspase3,9 signaling pathway. TP53 in the downstream of the EP300 / TP53 / Hippo / WNT signaling pathway can also induce cardiomyopathy and hypertension-induced CVD by suppressing the Hippo and WNT pathways (19). The SUMO1 gene through the TLR4 / STAT3 / SUM01 / Caspase-1 pathway can affect inflammation induction in EC. Following induction of inflammation in EC, EC dysfunction occurs, resulting in CVD due to hypertension. In addition, the JAK2 / STAT3 pathway in the upstream signaling of the SUMO1 gene can lead to cholesterol synthesis.

Cholesterol also increases sodium uptake and induces hypertension by increasing the function of the Furin molecule, as well as by the 4HNE / SGK1 / Nedd4-2 / ENaC pathway $(20,21)$. In a study by Yao et al., the results showed that the signaling pathway induced by the SUMO1 gene was effective in the development of hypertension(22). The KDR gene can induce vascular angiogenesis through the BCL6B / KLF4 / EGR1 / KDR / PI3K / AKT signaling pathway. Consequently, vascular angiogenesis is effective in the development of hypertension-induced CVD.

In addition, the PI3K / AKT pathway in the downstream of KDR signaling can inhibit the activity of Plasminogen Activator Inhibitor type1 (PAI-1), and thus affects plasminogen activity induction.

Subsequently, activated plasminogen in the downstream of the KDR signaling pathway can be converted to plasmin by tissue activator plasminogen (tPA). Plasmin activates the ERK1 / 2 and MAPK signaling pathways via the pro-BDNF / BDNF / TrKB pathway. Subsequently, the ERK1 / 2 signaling pathway activated in the downstream of plasmin activity can lead to Peripheral Arterial Smooth Muscle Cell (PASMC) proliferation via GATA4; it induces hypertension. 
Also, the MAPK signaling pathway increases the expression of adhesion molecules and ET-1, and decreases NO expression by decreasing PI3K / eNOS signaling. Hypertension is also a consequence of increased expression of ET-1 and adhesion molecules due to resistance arterioles(23, 24). In the study by Eyries et al., the findings showed, that KDR heterozygous loss of function plays an important role in the development of familial hypertension (25). The SNRPD3 gene through the SUN2 / SNRPD3 / MAU2 / ESPL1 / SMC1 and SNRPD3 / SMC1 / NF-K $\beta$ pathways can induce angiogenesis and EC dysfunction by inducing proliferation and inflammation in ECs, respectively.

The consequence of angiogenesis and EC dysfunction induced by the SNRPD3 gene is the incidence of CVD induced by hypertension. In addition, the SNRPD3 gene in conjunction with the MDM2 gene could play a negative role in p53 molecule regulation $(26,27)$. Subsequently, the MDM2 molecule induces proliferation in EC by suppressing the P53 signaling pathway, which results in the induction of hypertension. MDM2 can also activate the Angiotensin Converting Enzyme (ACE). The ACE2 enzyme subsequently converts Angiotensin 1-7 to Angiotensin II. Angiotensin II can also induce oxidative stress in EC via the Angll / AT1R / P38MAPK / MK2 / P47 / P22 / NOX / rac / P67 / ROS, which results in cell injury, EC dysfunction and hypertension.

On the other hand, MDM2 in the downstream of the PHLPP1 / Nrf2 / MdM2 / FOXO1 signaling pathway can also lead to renal cell apoptosis. It is also effective in inducing hypertension through damage to kidney cells and defects in the function of the renin-angiotensin system. In addition, the MDM2 molecule can increase the contraction of cardio myocytes in the downstream of the GRK2 / MDM2 / $\beta$-adrenergic receptor signaling pathway, and subsequently affects hypertension and CVD occurrence(28).

The DERL2 gene is responsible for the synthesis of the Derlin 2 protein. Subsequently, the Derlin-2 protein via the HRD1 / Shh / PTCH1 / SMO / GLi pathway can induce proliferation in arterial VSMCs. Induction of proliferation in arterial VSMCs is associated with angiogenesis and subsequent induction of hypertension-induced CVD. In addition, the Shh / YAP / Wnt / $\beta$-Catenin pathway in the downstream of the signaling of the DERL2 gene can lead to the transcription of the erythropoietin gene and its production. Erythropoietin is also effective in the synthesis of hemoglobin. Hemoglobin can induce vasoconstriction and subsequent hypertension by suppressing the cGmp / eNOS / NO / GC / PKG signaling pathway $(29,30)$.

The VCP gene can induce inflammation in the EC via the PINK1 / UFD / NPL4 / GP78 / HDR1 / VCP / NF$\mathrm{K} \beta$ signaling pathway. In addition, hypertension through the VCP / mTORC1 / Raptor / mTOR / mLST8 and VCP / mTORC2 / Rictor / mTOR / mLST8 pathways can be effective in inducing myocardial hypertrophy. Also in the P97 / VCP / KBTBD7 / Wnt / PCR / MARCH6 / Vangl pathway, the MARCH6 molecule is activated in the downstream of VCP signaling. Subsequently, the MARCH6 molecule involved in post-translational changes and production of the Lanosterol Synthase (LSS) enzyme(31, 32).

LSS enzyme converts Squalene 2,3-epoxide to Lanosterol. The lanosterol produced by the LSS enzyme is also converted to 7-Dehydrocholesterol (7DHC) by the enzyme Sterol-C5desturase (SC5D) in the pathway of cholesterol synthesis. Subsequently, 7DHC can induce atherogenesis and atherosclerosis by 
suppressing the TGF- $\beta$ signaling pathway. The consequence of atherogenesis and atherosclerosis increases blood pressure through vascular stiffness (33). The EIF4B gene activates C / EBP- $\beta$ / LAP signaling via the RSK / elF4B pathway. Consequently, the C / EBP- $\beta$ pathway in the downstream of EIF4B gene signaling can be effective in inducing hypertension. Thus, downstream of the Activating Transcription 4 / C-EBP- $\beta$ signaling pathway, the EBP protein can lead to cellular apoptosis by inducing endoplasmic stress in ECs, and subsequently induces hypertension due to EC dysfunction. In addition, in the downstream of the CXCR7 / P38 / C-EBP- $\beta$ signaling pathway, EBP can lead to EC senescence and inflammation in the ECs (34-36).

The NOTCH1 gene can be involved in inducing angiogenesis and apoptosis in EC through the Notch1 / NICD / RPBJ / Hey 1 / Hes1 and Notch1 / NICD / PI3K / $\beta$-Cat / TCF signaling pathways. Mutations in the NOTCH1 gene lead to increased expression of the GAB1 gene. Increased expression of GAB1 gene in the downstream of the PGE4 / EP4R / EGFR / Gab1 / Akt / NF-KB / VEGF signaling pathway can lead to hypertension by inducing angiogenesis in $\operatorname{ECs}(37,38)$.

On the other hand, Gab1 can inhibit Angiotensin II induced atherosclerosis through the HGF / GMet signaling pathway, thereby inhibiting the induction of hypertension-induced CVD. Thus, the Gab1 molecule has dual effects on the incidence of CVD, due to hypertension and can act as a double-edged sword (39). In a study by Wang et al., the results showed that the NOTCH1 gene signaling pathway could be effective in the development of hypertension (40). In addition, the MAML1 gene in the downstream of the Notch1 signaling pathway through Notch / NEC / HD / ICN / MAML1 / CFB1 can induce proliferation in arterial VSMCs. Induction of proliferation in arterial VSMCs is associated with vascular angiogenesis and subsequent hypertension. The Notch / MAML1 pathway can also lead to upregulation of the RASA1 gene by suppressing the ERK / Kras signaling. Subsequently, RASA1 can also induce inflammation in the EC via the RASA1 / Ras / MAPK / MK2 signaling pathway (41-43). Also in the study of Dabral et al., the findings indicated that MAML1 in the downstream of the NOTCH1 signaling pathway could be effective in proliferation regulating and induction of apoptosis in ECs, and subsequently in the development of hypertension due to endothelial dysfunction (44).

The SOCS3 gene, through the STAT3 / P53 / P21 pathway can induce apoptosis in EC. Following induction of apoptosis in these cells by the SOCS3 signaling pathway, EC dysfunction occurs, resulting in hypertension-induced CVD. In addition, the STAT3 molecule in the downstream of the SOCS3 gene signaling pathway can bind the SOCS3 gene signaling to the ROCK1 gene via ROCK1 / JAK2 / STAT3. Subsequently, the RhoA / ROCK1 / myosin phosphatase signaling pathway targeting subunit / myosin light chain can lead to angiogenesis and subsequent hypertension by inducing proliferation in VSMC. The ROCK1 / SP1 / PKC-y pathway can also induce hypertension due to hypertrophy in cardio myocytes (45, 46).

In the study of Kuang et al., the results showed that polymorphism in the SOCS3 gene is associated with hypertension occurrence (47). The CBL gene can induce proliferation in the EC via the Raf / Ras / MAPK signaling pathway. Subsequently, by inducing proliferation in the EC, angiogenesis occurs and the 
consequence of CVD is hypertension. In addition, activation of the MAPK molecule in the downstream of the CBL gene signaling pathway via the Raf / MEK / P42 / 44 MAPK / P90RSK / GSK can increase LDL receptor expression on the EC surface, leading to increased intravascular cholesterol deposition; thereby, it induces atherosclerosis and hypertension. On the other hand, the MEK1 / P42 / 44 MAPK signaling pathway can protect cardio myocytes against damage caused by heart reperfusion. Therefore, the MAPK signaling pathway has dual effects on the occurrence of hypertension, as well as CVD risk reduction; it also acts like a double-edged sword(48).

The CBL gene can also lead to upregulation of the KRAS molecule via the KRAS / HRAS / NRAS / BRAF / RAF-1 / SHOC2 / MEK1,2 / ERK1,2 pathway. KRAS also affects hypertension, cardiac hypertrophy and subsequent CVD through RAF / MEK1 / 2 / ERK1 / 2 and PI3K / Akt / mTOR signaling pathways(49). Also the ICAM-1 gene in the downstream of the TNF-a / TNFRI / c-Src / NADPH oxidase / ROS / NF-K $/$ ICAM-1 signaling pathway can induce inflammation in EC. The ICAM-1 gene can also communicate with VEGFA gene signaling via the VEGFA / TNF-a / ICAM-1 pathway. Subsequently, the VEGFA molecule can induce vascular angiogenesis by inducing Akt / PKE / ERK1 / 2 and Src / FAK / RhoGTPase signaling. VEGFA is also activated in the downstream of the TSP1 / NF-K $/$ VEGFA pathway and may affect occurrence of angiogenesis-induced hypertension and CVD(50-52).

In the study of Ataam et al., the findings indicated the role of ICAM-1 gene in the development of hypertension, following pulmonary thromboembolism (53). The ITGB2 gene is responsible for the synthesis of Integrin- $\beta 2$. Subsequently, it can induce proliferation in ECs by beta integrin synthesis, and induction of the FAK / mTORC1 pathway in the downstream of this molecule. Also, the mTORC1 molecule in the downstream of the ITGB2 gene signaling pathway can bind the ITGB2 gene signaling to the TRAF6 gene, through the mTORC1 / Raptor / LST8 / GßL / S6K / MyD88 / TRAF6 signaling. The product of the TRAF6 gene in the downstream signaling of the ITGB2 gene is ubiquitin E3 ligase. This molecule can also lead to cardiac hypertrophy and subsequent hypertension via the TRAF6 / TAK1 / MAPK / Nrf2 signaling pathway $(54,55)$.

The results of a study by Sun et al. showed that polymorphisms in the ITGB2 gene can be associated with coronary heart disease, and finally hypertension (56). STRN gene can lead to proliferation in ECs by inducing STK25 / STRN / AMPK / ACC1 signaling. Proliferation induction in EC by vascular angiogenesis is effective in the development of CVD due to hypertension. Also, the Ca MKK2 / AMPK / Pref-1 / SOX-9 pathway in the downstream of the STRN gene signaling can induce the TLR4 signaling pathway, through the CaMKK2 molecule. Consequently, the TLR4 signaling pathway can be effective in myocardial hypertrophy and hypertension via the TLR4 / TRIF / Peli1 / RIP1 / IKK / NF-Kß $(57,58)$. The MEF2A gene downstream of the Tp12 / MEK5 / ERK5 / BMK1 / MEF2A signaling pathway can induce apoptosis in ECs.

Also, the ERK5 molecule in the upstream signaling of the MEF2A gene can lead to the binding of the MEF2A gene signaling to the RAC1 gene, via the RAC1 / MEK / ERK pathway. Subsequently, RAC1 in the downstream of the Ang II / AT1R / PKCY / RAC1 signaling pathway can play a role in inducing 
hypertension by activating the sympathetic nerves. Furthermore, RAC1 can be effective in inducing oxidative stress, and subsequent cell damage in ECs by suppressing the AMPK / ERK1 / 2 / RSK / NOS, and activating the NADPH oxidase / ROS pathway. The consequence of these processes is the induction of EC dysfunction, and the occurrence of hypertension(59-61).

The PTPRC gene can also induce proliferation in arterial VSMCs via the BCR / ABL / ERK / ARG / TWIST1 / ZEB1 signaling pathway. By inducing proliferation in arterial VSMCs, the phenomenon of vascular angiogenesis occurs, which results in the development of CVD due to hypertension(62). In addition, in a study by Esmael et al., the findings suggested that the MEF2A and PTPRC genes were associated with coronary artery disease and subsequent hypertension(63).

\section{microRNAs}

Important microRNAs, that involved in the development of hypertension-induced CVD based on the bioinformatics findings of our study are:

miR-3135-3P, miR-505, miR-6088, miR-451, miR-4637-3P, miR-1281-3P, miR-4304-3P, miR-323-3P, miR3150-3p.1, miR-4718, miR-151-5p, miR-3117-5p.2, miR-3613, miR-1278- 3P, miR-4467, miR-4730, miR190b-3p, miR-610-5p, miR-3648-3p, miR-4796-3p, miR-2277-3P, miR-325, miR-6807-3p, miR-551a-3p, and miR-3131-3P.

miR-3135-3p via the GOLPH3 / AKT1 / mTOR pathway can induce apoptosis in ECs. miR-3135-3P can also lead to EC dysfunction, and consequently induces hypertension by reducing the expression of HIF-1a and TWIST1 molecules, and increases the expression of ET-1 molecules in the downstream of the ERK / MAPK signaling pathway(64). miR-505 can inhibit proliferation in the VSMC by suppressing the PI3K / Akt / GSK3 $\beta$ / Cyclin D1 pathway, and reducing the expression of proliferating cell nuclear antigen (PCNA); subsequently it leads to hypertension in individuals with atherosclerosis(65). Also, miR-505-3P via the SIRT3 / ROS / NET pathway can exacerbate atherosclerosis, and subsequently induces hypertension due to vascular stiffness (66). In a study by Escate et al., the results showed that miR-505 plays a significant role in inducing atherosclerosis, due to familial hypercholesterolemia and subsequent hypertension via regulating the expression of chemokine receptors and upregulation of inflammationrelated signaling pathways(67).

On the other hand, miR-505-3P can reduce vasoconstriction, and subsequently prevents hypertension by suppressing the NCX1 pathway; it also reduces $\mathrm{Ca}^{+2}$ overload. Also, by reducing $\mathrm{Ca}^{+2}$ overload in cardio myocytes, miR-505-3P can prevent the induction of Bax / CytochromeC / Caspase3,9 pathway by targeting the TP53AIP1 molecule, which leads to apoptosis in heart muscle cells; therefore, it reduces the incidence of CVD (68). miR-6088 can induce proliferation in vascular ECs by suppressing the SMARCB / INI1 / Snf5 signaling pathway (69). miR-451 induces proliferation in arterial smooth muscle cells by suppressing the KLLN / P53 and CAB39 / LKB1 / AMPK / mTORC1 / S6K pathways, leading to hypertension by reducing vessel diameter and increasing vascular thickness(70). 
In a study by Gu et al., the results showed that miR-451 could affect development of hypertension following myocardial ischemia reperfusion injury by regulating the AMPK signaling pathway(71). miR4637-3p can induce inflammation in EC by inhibiting the activity of the IFN-I molecule, and inducing the Keap1 / Nrf2 / HO-1 / NQ01 / GST / ROS / TXNIP / NLRP3 / IL-1 $\beta$ pathway. The ROS molecule in the downstream of the miR-4637-3p signaling pathway can also lead to apoptosis in ECs by inducing the PUMA / NOXA / BAX / Caspase3,9 / PARP pathway.

In addition, miR-4637-3p may affect apoptosis induction in cardio myocytes, and subsequent CVD due to hypertension, as a result of myocardial hypertrophy and via suppressing the ErbB / PI3K signaling pathway (72). miR-1281-3p in the PI3K / DNMT1 / miR-1281-3p / HDAC4 / PDGF-BB pathway could lead to increased proliferation in VSMCs. The consequence of increased proliferation in VSMCs by miR-1281$3 p$ is induction of angiogenesis and subsequent hypertension. In addition, miR-1281-3p can induce proliferation in VSMC by downstream of the P65NF-K $\beta$ / miR-455 / PTEN signaling pathway and suppressing the PTEN molecule; finally, this process leads to hypertension and CVD due to vascular stenosis(73).

miR-4304-3p can induce proliferation in VSMC by suppressing the p38MAPK / HIF / MEF2C and HIF-1a / CTGF / PAI-1 signaling pathways, and subsequently induces hypertension through vascular angiogenesis (74). miR-323-3P can induce proliferation in ECs by regulating the TMEFF2 / AKT / ERK, NPTX1 / Akt and BTG1 / ERK / MEK signaling pathways. Following proliferation in ECs, the phenomenon of angiogenesis occurs, which results in the onset of CVD due to hypertension (75).

In a study by Pilbrow et al., the results showed that miR-323-3p could be used as a diagnostic biomarker for progression of acute coronary syndrome(76). In addition, a study by Cervera et al. showed, that miR323-3p can be used as an indicator in the diagnosis of hypertension following cardiomyopathy(77). miR3150-3p.1 can inhibit the induction of cellular apoptosis by increasing the expression of VEGF, VEGFA, FLT1 / 2 and FOXO molecules in the downstream of the LRIG1 / ERGF / PI3K / Akt signaling pathway, and subsequently induces proliferation in the EC(78). miR-4718 leads to stenosis in the carotid arteries by targeting the PLCY / DAG / PKC / Ca+2 / DAG / GEF / RAP1 / Talin-1 signaling pathway. In addition, miR4718 can induce apoptosis in EC by targeting the HER2 / PI3K / PDK / Akt / IAP / XIAP / CASP3 pathway; ultimately, it leads to CVD due to hypertension through EC dysfunction (79).

miR-151-5p can increase vasoconstriction and fibrosis in cardio myocytes; it subsequently induces hypertension by suppressing the YAP / SRECA2a / STAT3 / FOXM1 pathway, and increases the SNON / SK1 signaling(80). In a study by Zhang et al., the results showed that miR-151 could induce calcification in blood vessels by inhibiting the expression of the Atg molecule in VSMCs; it can lead to hypertension (81). miR-3117-5P.2 in the downstream of the YAP / TAT / OCT4 pathway can also lead to proliferation in EC by activating the PPARY / MAPK / APOE / LRP8 / COL1a1 / COL3a1 / LOX pathway; it affects angiogenesis-induced hypertension (82). 
miR-3613 can inhibit cell proliferation and induces apoptosis in cells by suppressing the KDM2A / PFKFB3 and CALB1 / D28K signaling pathways. In addition, miR-3613 can induce apoptosis in ECs by suppressing the PDK3 / Akt / Bad / Bcl-xl / BCL2 pathway, and inducing the Ctyo-c / Apaf1 / Caspase3,9 signaling (83). miR-1278-3P can inhibit cell proliferation and induces cell apoptosis by targeting the LRH1 / KIF5B / Wnt- $\beta$ Catenin signaling pathway; it can also regulate the BTG2 / JNK / NF-K $\beta$ pathway (84). miR-4467 can activate Caspase1,3 enzymes by regulating the signaling pathway of SF1 / TGF $\beta R 2$ / TGF $\beta$ / Smad2,3 /Bim, which can induce apoptosis in ECs by leaving cytochrome C, as well as by ROSinduced inflammasome activation (85).

miR-4730 can induce apoptosis and inflammation in cardio myocytes by regulating the signaling pathways of MAPK3 / SIRT1 / PGC1 a and PARP-1 / NF-K $\beta$, and subsequently affect CVD development due to hypertension (86). miR-190b-3p can inhibit cell proliferation and induce apoptosis in ECs by regulating the signaling pathway of TGF- $\beta$ / PI3K / Akt and TGF- $\beta$ / JNK / ERK1,2. miR-190b-3p can also be effective in inducing hypertension by regulating the Ang II / AT1R / TSP-1 / TGF- $\beta$ signaling pathway $(87,88)$. In addition, in a study by Jiang et al., the findings suggested that miR-190 could affect hypertension induction following hypoxia, by regulating the HIF-1a / KLFI5 pathway(89).

miR-610-5p can inhibit cell proliferation induction, and subsequently induces apoptosis in EC by suppressing the MEK / ERK / EIK1 / TWIST1 Raf-1 pathway. In addition, miR-610-5p can induce inflammation in ECs by suppressing the TWIST1 / Dnmt1 / miR-186 / NF-K $\beta$ pathway(90). In a study by Miao et al., the results showed that an increase in miR-610 levels is associated with hypertension following chronic thromboembolism (91).

In addition, miR-3648-3P can lead to cell proliferation in ECs by suppressing the APC2 molecule, and inducing Wnt / $\beta$-Catenin and IGF-1 / Akt signaling pathways, thereby inducing angiogenesis-induced hypertension (92). miR-4796-3p can also lead to endothelial dysfunction, hypertrophy and hypertension by suppressing the PTEN / AKT / FOXO3 and $\mathrm{ONOO}^{-}$/ Sirt1 / YAP signaling pathways(93).

miR-2277-3p can induce cell proliferation in ECs by suppressing the NUPR1L / DACH1 / Smad4 / Smad2 / 3 / SIX1 / EYA signaling pathway(94). miR-325 can also inhibit cell proliferation by suppressing the BAG / ERK1,2 pathway. In addition, downregulation of miR-325 through upregulation of IncRNA MEG3 and induction of the TRPV4 / Rho / ROCK pathway can lead to cardio myocyte damage; finally, it affects hypertension. In a study by Lazar et al., the results showed that miR-325 plays an important role in hypertension, and the incidence of PE in pregnant women $(95,96)$.

miR-6807-3p can also induce angiogenesis through downregulation of the eNOS enzyme, decreased NO production, increased VEGF expression and suppression of the DKK1 molecule in the downstream of the hub / Wnt / $\beta$-Catenin signaling pathway. Induction of angiogenesis is followed by exacerbated hypertension due to atherosclerosis(97). miR-551a-3p can be effective in inducing cell apoptosis in ECs, and subsequent hypertension by modulating the TRAF-6 / Erk / c-Fox / NFAT1 / NF-K $\beta$ and ROS / JNK / BCL2 / Beclin1 signaling pathways (98). miR-3131-3P can also prevent foam cell formation by regulating 
the expression of SCARB2, NCEHI1 and ABCG4 genes; it also affects hypertension induction, following coronary artery disease in the downstream of the Angll / ST2 / miR-202 / IL-13 / arginase 2 / Akt signaling pathway(99).

\section{Conclusion}

Identification of signaling pathways, genes and microRNAs involved in the development of CVD by hypertension is applicable in designing target therapy, to treat and reduce the risk of CVD following hypertension in pregnant women with PE. It is also very useful to study the up and downregulation of these pathways, genes and microRNAs in severe and non-severe PE patients; it also helps to identify diagnostic biomarkers. On the other hand, some pathways, genes and microRNAs act like double-edged swords, and more studies are needed to evaluate them.

\section{Declarations}

\section{Acknowledgements:}

We wish thank you of all our colleague in IRAN university of medical science.

\section{Authors' contributions:}

Sh.A and F.D has conceived the manuscript and revised it. M, P. and M. A wrote the manuscript. Sh.Gh and M.P. analysis of data.

\section{Compliance with ethical standards}

\section{Conflict of interest:}

The authors declare that they have no conflict of interest.

\section{Data availability:}

This is a review study, and it is not an original. Data availability is corresponding author responsibility.

\section{Ethical approval}

This article does not contain any studies with human participants or animals performed by any of the authors. 


\section{References}

1. Reyes LM, García RG, Ruiz SL, Camacho PA, Ospina MB, Aroca G, et al. Risk factors for preeclampsia in women from Colombia: a case-control study. PloS one. 2012;7(7):e41622.

2. Poorolajal J, Jenabi E. The association between body mass index and preeclampsia: a metaanalysis. The Journal of Maternal-Fetal \& Neonatal Medicine. 2016;29(22):3670-6.

3. Baker AM, Klein RL, Moss KL, Haeri S, Boggess K. Maternal serum dyslipidemia occurs early in pregnancy in women with mild but not severe preeclampsia. American journal of obstetrics and gynecology. 2009;201(3):293. e1-. e4.

4. Granger JP, Alexander BT, Llinas MT, Bennett WA, Khalil RA. Pathophysiology of hypertension during preeclampsia linking placental ischemia with endothelial dysfunction. Hypertension. 2001;38(3):71822.

5. Sibai BM. Diagnosis and management of gestational hypertension and preeclampsia. Obstetrics \& Gynecology. 2003;102(1):181-92.

6. Draghici S, Khatri P, Tarca AL, Amin K, Done A, Voichita C, et al. A systems biology approach for pathway level analysis. Genome research. 2007;17(10):1537-45.

7. Kang S, Dong S, Gu T-L, Guo A, Cohen MS, Lonial S, et al. FGFR3 activates RSK2 to mediate hematopoietic transformation through tyrosine phosphorylation of RSK2 and activation of the MEK/ERK pathway. Cancer cell. 2007;12(3):201-14.

8. Faustman DL, Davis M. TNF receptor 2 and disease: autoimmunity and regenerative medicine. Frontiers in immunology. 2013;4:478.

9. Xiong S, Hong Z, Huang LS, Tsukasaki Y, Nepal S, Di A, et al. IL-1 $\beta$ suppression of VE-cadherin transcription underlies sepsis-induced inflammatory lung injury. The Journal of clinical investigation. 2020;130(7):3684-98.

10. Koga H, Sugiyama S, Kugiyama K, Watanabe K, Fukushima H, Tanaka T, et al. Elevated levels of VEcadherin-positive endothelial microparticles in patients with type 2 diabetes mellitus and coronary artery disease. Journal of the American College of Cardiology. 2005;45(10):1622-30.

11. Xiao D, Huang $X$, Zhang L. Chronic hypoxia differentially up-regulates protein kinase C-mediated ovine uterine arterial contraction via actin polymerization signaling in pregnancy. Biology of reproduction. 2012;87(6):142, 1-9.

12. Kassem KM, Ali M, Rhaleb N-E. Interleukin 4: its role in hypertension, atherosclerosis, valvular, and nonvalvular cardiovascular diseases. Journal of cardiovascular pharmacology and therapeutics. 2020;25(1):7-14.

13. Yang $M$, Song L, Wang L, Yukht A, Ruther H, Li F, et al. Deficiency of GATA3-positive macrophages improves cardiac function following myocardial infarction or pressure overload hypertrophy. Journal of the American College of Cardiology. 2018;72(8):885-904.

14. Forrester SJ, Kawai T, O'Brien S, Thomas W, Harris RC, Eguchi S. Epidermal growth factor receptor transactivation: mechanisms, pathophysiology, and potential therapies in the cardiovascular system. 
Annual review of pharmacology and toxicology. 2016;56:627-53.

15. Papparella I, Ceolotto G, Montemurro D, Antonello M, Garbisa S, Rossi G, et al. Green tea attenuates angiotensin Il-induced cardiac hypertrophy in rats by modulating reactive oxygen species production and the Src/epidermal growth factor receptor/Akt signaling pathway. The Journal of nutrition. 2008;138(9):1596-601.

16. He K, Sun H, Zhang J, Zheng R, Gu J, Luo M, et al. Rab7-mediated autophagy regulates phenotypic transformation and behavior of smooth muscle cells via the Ras/Raf/MEK/ERK signaling pathway in human aortic dissection. Molecular Medicine Reports. 2019;19(4):3105-13.

17. Yin H, Li Q, Qian G, Wang Y, Li Y, Wu G, et al. Rab1 GTPase regulates phenotypic modulation of pulmonary artery smooth muscle cells by mediating the transport of angiotensin II type 1 receptor under hypoxia. The international journal of biochemistry \& cell biology. 2011;43(3):401-8.

18. Wu G, Yussman MG, Barrett TJ, Hahn HS, Osinska H, Hilliard GM, et al. Increased myocardial Rab GTPase expression: a consequence and cause of cardiomyopathy. Circulation research. 2001;89(12):1130-7.

19. Hampp S, Kiessling T, Buechle K, Mansilla SF, Thomale J, Rall M, et al. DNA damage tolerance pathway involving DNA polymerase $ı$ and the tumor suppressor p53 regulates DNA replication fork progression. Proceedings of the National Academy of Sciences. 2016;113(30):E4311-E9.

20. Li J, Zhou Y, Liu Y, Dai B, Zhang Y-H, Zhang P-F, et al. Sorafenib inhibits caspase-1 expression through suppressing TLR4/stat3/SUM01 pathway in hepatocellular carcinoma. Cancer biology \& therapy. 2018;19(11):1057-64.

21. Xu X, Han K, Zhu J, Mao H, Lin X, Zhang Z, et al. An inhibitor of cholesterol absorption displays antimyeloma activity by targeting the JAK2-STAT3 signaling pathway. Oncotarget. 2016;7(46):75539.

22. Yao Y, Li H, Da X, He Z, Tang B, Li Y, et al. SUMOylation of Vps34 by SUMO1 promotes phenotypic switching of vascular smooth muscle cells by activating autophagy in pulmonary arterial hypertension. Pulmonary pharmacology \& therapeutics. 2019;55:38-49.

23. Wang T, Guo Y, Liu S, Zhang C, Cui T, Ding K, et al. KLF4, a Key Regulator of a Transitive Triplet, Acts on the TGF- $\beta$ Signaling Pathway and Contributes to High-Altitude Adaptation of Tibetan Pigs. Frontiers in genetics. 2021;12.

24. Mukai Y, Wang C-Y, Rikitake Y, Liao JK. Phosphatidylinositol 3-kinase/protein kinase Akt negatively regulates plasminogen activator inhibitor type 1 expression in vascular endothelial cells. American Journal of Physiology-Heart and Circulatory Physiology. 2007;292(4):H1937-H42.

25. Eyries M, Montani D, Girerd B, Favrolt N, Riou M, Faivre L, et al. Familial pulmonary arterial hypertension by KDR heterozygous loss of function. European Respiratory Journal. 2020;55(4).

26. Koedoot E, van Steijn E, Vermeer M, González-Prieto R, Vertegaal AC, Martens JW, et al. Splicing factors control triple-negative breast cancer cell mitosis through SUN2 interaction and sororin intron retention. Journal of Experimental \& Clinical Cancer Research. 2021;40(1):1-17.

27. Czarnek M, Sarad K, Karaś A, Kochan J, Bereta J. Non-targeting control for MISSION shRNA library silences SNRPD3 leading to cell death or permanent growth arrest. Molecular Therapy-Nucleic Acids. 
2021;26:711-31.

28. Jean-Charles P-Y, Yu SM-W, Abraham D, Kommaddi RP, Mao L, Strachan RT, et al. Mdm2 regulates cardiac contractility by inhibiting GRK2-mediated desensitization of $\beta$-adrenergic receptor signaling. JCl insight. 2017;2(17).

29. Huang C-H, Hsiao H-T, Chu Y-R, Ye Y, Chen X. Derlin2 protein facilitates HRD1-mediated retrotranslocation of sonic hedgehog at the endoplasmic reticulum. Journal of Biological Chemistry. 2013;288(35):25330-9.

30. Riou R, Ladli M, Gerbal-Chaloin S, Bossard P, Gougelet A, Godard C, et al. ARID1A loss in adult hepatocytes activates $\beta$-catenin-mediated erythropoietin transcription. Elife. 2020;9:e53550.

31. Powell SR, Herrmann J, Lerman A, Patterson C, Wang X. The ubiquitin-proteasome system and cardiovascular disease. Progress in molecular biology and translational science. 2012;109:295-346.

32. Feng D, Wang J, Yang W, Li J, Lin X, Zha F, et al. Regulation of Wnt/PCP signaling through p97/VCPKBTBD7-mediated Vangl ubiquitination and endoplasmic reticulum-associated degradation. Science advances. 2021;7(20):eabg2099.

33. Huang SS, Liu IH, Chen CL, Chang JM, Johnson FE, Huang JS. 7-Dehydrocholesterol (7-DHC), But Not Cholesterol, Causes Suppression of Canonical TGF-BSignaling and Is Likely Involved in the Development of Atherosclerotic Cardiovascular Disease (ASCVD). Journal of cellular biochemistry. 2017;118(6):1387-400.

34. Huber R, Panterodt T, Welz B, Christmann M, Friesenhagen J, Westphal A, et al. C/EBPß-LAP*/LAP expression is mediated by RSK/elF4B-dependent signalling and boosted by increased protein stability in models of monocytic differentiation. PLoS One. 2015;10(12):e0144338.

35. Liu X, Huang Z, Zhang Y, Shui X, Liu F, Wu Z, et al. Lacidipine Ameliorates the Endothelial Senescence and Inflammatory Injury Through CXCR7/P38/C/EBP- $\beta$ Signaling Pathway. Frontiers in Cardiovascular Medicine. 2021:634.

36. Chen W, Liu T, Liang Q, Chen X, Tao W, Fang M, et al. miR-1283 Contributes to Endoplasmic Reticulum Stress in the Development of Hypertension Through the Activating Transcription Factor-4 (ATF4)/C/EBP-Homologous Protein (CHOP) Signaling Pathway. Medical Science Monitor: International Medical Journal of Experimental and Clinical Research. 2021;27:e930552-1.

37. Zang M-d, Hu L, Fan Z-y, Wang H-x, Zhu Z-I, Cao S, et al. Luteolin suppresses gastric cancer progression by reversing epithelial-mesenchymal transition via suppression of the Notch signaling pathway. Journal of translational medicine. 2017;15(1):1-11.

38. Zheng Y, Wang Z, Ding X, Zhang W, Li G, Liu L, et al. A novel Notch1 missense mutation (C1133Y) in the Abruptex domain exhibits enhanced proliferation and invasion in oral squamous cell carcinoma. Cancer Cell International. 2018;18(1):1-15.

39. Higuchi K, Nakaoka Y, Shioyama W, Taniyama Y, Arita Y, Hashimoto T, et al. Gab1 Attenuates Angiotensin II-accelerated Atherosclerosis in Apolipoprotein E Deficient Mice via HGF/c-Met Signaling Pathway. Am Heart Assoc; 2010. 
40. Wang S, Zhu G, Jiang D, Rhen J, Li X, Liu H, et al. Reduced Notch1 Cleavage Promotes the Development of Pulmonary Hypertension. Hypertension. 2022;79(1):79-92.

41. Pancewicz J, Nicot C. Current views on the role of Notch signaling and the pathogenesis of human leukemia. BMC cancer. 2011;11(1):1-7.

42. Kong G, You X, Wen Z, Chang Y-I, Qian S, Ranheim EA, et al. Downregulating Notch counteracts KrasG12D-induced ERK activation and oxidative phosphorylation in myeloproliferative neoplasm. Leukemia. 2019;33(3):671-85.

43. Kent OA, Mendell JT, Rottapel R. Transcriptional regulation of miR-31 by oncogenic KRAS mediates metastatic phenotypes by repressing RASA1. Molecular Cancer Research. 2016;14(3):267-77.

44. Dabral S, Tian X, Kojonazarov B, Savai R, Ghofrani HA, Weissmann N, et al. Notch1 signalling regulates endothelial proliferation and apoptosis in pulmonary arterial hypertension. European Respiratory Journal. 2016;48(4):1137-49.

45. Chen J, Pan J, Wang J, Song K, Zhu D, Huang C, et al. Soluble egg antigens of Schistosoma japonicum induce senescence in activated hepatic stellate cells by activation of the STAT3/p53/p21 pathway. Scientific reports. 2016;6(1):1-11.

46. Oh RS, Haak AJ, Smith KM, Ligresti G, Choi KM, Xie T, et al. RNAi screening identifies a mechanosensitive ROCK-JAK2-STAT3 network central to myofibroblast activation. Journal of cell science. 2018;131(10):jcs209932.

47. Kuang D, Dong L, Liu L, Zuo M, Xie Y, Li T, et al. SOCS3 Gene Polymorphism and Hypertension Susceptibility in Chinese Population: A Two-Center Case-Control Study. BioMed Research International. 2021;2021.

48. Hong Y, Keylock A, Jensen B, Jacques TS, Ogunbiyi O, Omoyinmi E, et al. Cerebral arteriopathy associated with heterozygous variants in the casitas B-lineage lymphoma gene. Neurology Genetics. 2020;6(4).

49. Roberts AE, Allanson JE, Tartaglia M, Gelb BD. Noonan syndrome. The Lancet. 2013;381(9863):33342.

50. Lee I-T, Luo S-F, Lee C-W, Wang S-W, Lin C-C, Chang C-C, et al. Overexpression of HO-1 protects against TNF-a-mediated airway inflammation by down-regulation of TNFR1-dependent oxidative stress. The American journal of pathology. 2009;175(2):519-32.

51. Thichanpiang P, Harper SJ, Wongprasert K, Bates DO. TNF-a-induced ICAM-1 expression and monocyte adhesion in human RPE cells is mediated in part through autocrine VEGF stimulation. Molecular Vision. 2014;20:781.

52. Guo M-L, Kook YH, Shannon CE, Buch S. Notch3/VEGF-A axis is involved in TAT-mediated proliferation of pulmonary artery smooth muscle cells: Implications for HIV-associated PAH. Cell death discovery. 2018;4(1):1-13.

53. Ataam JA, Mercier O, Lamrani L, Amsallem M, Ataam JA, Ataam SA, et al. RETRACTED: ICAM-1 promotes the abnormal endothelial cell phenotype in chronic thromboembolic pulmonary hypertension. Elsevier; 2019. 
54. Li H, Hu S, Pang Y, Li M, Chen L, Liu F, et al. Bufalin inhibits glycolysis-induced cell growth and proliferation through the suppression of Integrin $\beta 2 / F A K$ signaling pathway in ovarian cancer. American Journal of Cancer Research. 2018;8(7):1288.

55. Manicassamy S, Pulendran B, editors. Modulation of adaptive immunity with Toll-like receptors. Seminars in immunology; 2009: Elsevier.

56. Sun Y, Long-Wang T, Zeng Y, Gong F-Y, Zhu H-J, Pan H, et al. Association of SRB1, ITGB2 gene polymorphisms with coronary heart disease in Chinese Han population. bioRxiv. 2018:402792.

57. Zhang Y, Xu J, Qiu Z, Guan Y, Zhang X, Zhang X, et al. STK25 enhances hepatocellular carcinoma progression through the STRN/AMPK/ACC1 pathway. Cancer cell international. 2022;22(1):1-23.

58. Williams JN, Sankar U. CaMKK2 signaling in metabolism and skeletal disease: a new axis with therapeutic potential. Current osteoporosis reports. 2019;17(4):169-77.

59. Kim JM, Hwang I-H, Jang I-S, Kim M, Bang IS, Park SJ, et al. Houttuynia cordata Thunb promotes activation of HIF-1A-FOXO3 and MEF2A pathways to induce apoptosis in human HepG2 hepatocellular carcinoma cells. Integrative Cancer Therapies. 2017;16(3):360-72.

60. Nie T, Hui X, Mao L, Nie B, Li K, Sun W, et al. Harmine induces adipocyte thermogenesis through RAC1-MEK-ERK-CHD4 axis. Scientific reports. 2016;6(1):1-10.

61. Su Q, Huo C-J, Li H-B, Liu K-L, Li X, Yang Q, et al. Renin-angiotensin system acting on reactive oxygen species in paraventricular nucleus induces sympathetic activation via AT1R/PKCY/Rac1 pathway in salt-induced hypertension. Scientific reports. 2017;7(1):1-13.

62. Drube J, Ernst T, Pfirrmann M, Albert BV, Drube S, Reich D, et al. PTPRG and PTPRC modulate nilotinib response in chronic myeloid leukemia cells. Oncotarget. 2018;9(10):9442.

63. Abdu Esmael TS. Some Regulation Mechanisms of Candidate Genes for Human Cardiovascular Diseases. Journal Of Hypertension And Cardiology. 2021;3(1):28-36.

64. Núñez-Olvera SI, Chávez-Munguía B, del Rocío Terrones-Gurrola MC, Marchat LA, Puente-Rivera J, Ruíz-García E, et al. A novel protective role for microRNA-3135b in Golgi apparatus fragmentation induced by chemotherapy via GOLPH3/AKT1/mTOR axis in colorectal cancer cells. Scientific reports. 2020;10(1):1-14.

65. Tang H, Lv W, Sun W, Bi Q, Hao Y. miR-505 inhibits cell growth and EMT by targeting MAP3K3 through the AKT-NFKB pathway in NSCLC cells. International journal of molecular medicine. 2019;43(3):1203-16.

66. Chen L, Hu L, Li Q, Ma J, Li H. Exosome-encapsulated miR-505 from ox-LDL-treated vascular endothelial cells aggravates atherosclerosis by inducing NET formation. Acta biochimica et biophysica Sinica. 2019;51(12):1233-41.

67. Escate R, Mata P, Cepeda JM, Padreó T, Badimon L. miR-505-3p controls chemokine receptor upregulation in macrophages: role in familial hypercholesterolemia. The FASEB Journal. 2018;32(2):601-12.

68. Fang H, Liu Y, He Y, Jiang Y, Wei Y, Liu H, et al. Extracellular vesicle-delivered miR-505-5p, as a diagnostic biomarker of early lung adenocarcinoma, inhibits cell apoptosis by targeting TP53AIP1. 
International journal of oncology. 2019;54(5):1821-32.

69. Gong X, Huang M-Y. Tumor-suppressive function of IncRNA-MEG3 in glioma cells by regulating miR6088/SMARCB1 axis. BioMed Research International. 2020;2020.

70. Guo R, Gu J, Zhang Z, Wang Y, Gu C. MiR-451 promotes cell proliferation and metastasis in pancreatic cancer through targeting CAB39. BioMed research international. 2017;2017.

71. Guo Y, Gao J, Liu Y, Zhang X, An X, Zhou J, et al. miR-451 on Myocardial Ischemia-Reperfusion in Rats by Regulating AMPK Signaling Pathway. BioMed Research International. 2021;2021.

72. Yi X, Cheng X. Understanding Competitive Endogenous RNA Network Mechanism in Type 1 Diabetes Mellitus Using Computational and Bioinformatics Approaches. Diabetes, Metabolic Syndrome and Obesity: Targets and Therapy. 2021;14:3865.

73. Li Y, Li L, Qian Z, Lin B, Chen J, Luo Y, et al. Phosphatidylinositol 3-Kinase-DNA Methyltransferase 1-miR-1281-Histone Deacetylase 4 Regulatory Axis Mediates Platelet-Derived Growth FactorInduced Proliferation and Migration of Pulmonary Artery Smooth Muscle Cells. Journal of the American Heart Association. 2018;7(6):e007572.

74. Agrawal R, Dale TP, Al-Zubaidi MA, Benny Malgulwar P, Forsyth NR, Kulshreshtha R. Pluripotent and multipotent stem cells display distinct hypoxic miRNA expression profiles. PLoS One. 2016;11(10):e0164976.

75. Fan J-m, Zheng Z-r, Zeng Y-M, Chen X-y. MiR-323-3p targeting transmembrane protein with EGF-like and 2 follistatin domain (TMEFF2) inhibits human lung cancer A549 cell apoptosis by regulation of AKT and ERK signaling pathways. Medical science monitor: international medical journal of experimental and clinical research. 2020;26:e919454-1.

76. Pilbrow AP, Cordeddu L, Cameron VA, Frampton CM, Troughton RW, Doughty RN, et al. Circulating miR-323-3p and miR-652: candidate markers for the presence and progression of acute coronary syndromes. International journal of cardiology. 2014;176(2):375-85.

77. Seco-Cervera M, Gonzalez-Rodriguez D, Ibanez-Cabellos J, Peiró-Chova L, González-Cabo P, GarcíaLópez E, et al. Circulating miR-323-3p is a biomarker for cardiomyopathy and an indicator of phenotypic variability in Friedreich's ataxia patients. Scientific reports. 2017;7(1):1-12.

78. Schwarzenbacher D, Klec C, Pasculli B, Cerk S, Rinner B, Karbiener M, et al. MiR-1287-5p inhibits triple negative breast cancer growth by interaction with phosphoinositide 3-kinase $C B$, thereby sensitizing cells for PI3Kinase inhibitors. Breast Cancer Research. 2019;21(1):1-15.

79. Quintanilha JC, Cursino MA, Borges JB, Torso NG, Bastos LB, Oliveira JM, et al. MiR-3168, miR-6125, and miR-4718 as potential predictors of cisplatin-induced nephrotoxicity in patients with head and neck cancer. BMC cancer. 2021;21(1):1-10.

80. Liu X, Su X, Xu S, Wang H, Han D, Li J, et al. MicroRNA in vivo precipitation identifies miR-151-3p as a computational unpredictable miRNA to target Stat3 and inhibits innate IL-6 production. Cellular \& molecular immunology. 2018;15(2):99-110.

81. Chen L, Zhang R, Li J, Gao Y, Mao S. Exosomal miR-151-3p Induces Calcium Deposition in Vascular Smooth Muscle Cells by Inhibiting Atg5. 2021. 
82. Gutierrez-Camino A, Martin-Guerrero I, Dolzan V, Jazbec J, Carbone-Bañeres A, de Andoin NG, et al. Involvement of SNPs in miR-3117 and miR-3689d2 in childhood acute lymphoblastic leukemia risk. Oncotarget. 2018;9(33):22907.

83. Chen C, Pan Y, Bai L, Chen H, Duan Z, Si Q, et al. MicroRNA-3613-3p functions as a tumor suppressor and represents a novel therapeutic target in breast cancer. Breast Cancer Research. 2021;23(1):1-13.

84. Lin W, Zou H, Mo J, Jin C, Jiang H, Yu C, et al. Micro1278 Leads to Tumor Growth Arrest, Enhanced Sensitivity to Oxaliplatin and Vitamin D and Inhibits Metastasis via KIF5B, CYP24A1, and BTG2, Respectively. Frontiers in Oncology. 2021;11:666.

85. Shou J, Gu S, Gu W. Identification of dysregulated miRNAs and their regulatory signature in glioma patients using the partial least squares method. Experimental and therapeutic medicine. 2015;9(1):167-71.

86. Islam MT. Persistent epigenetic modulation by radiation exposure/insults in mammalian cells. Brazilian Journal of Biological Sciences. 2018;5(10):589-98.

87. Yu Y, Luo W, Yang Z-J, Chi J-R, Li Y-R, Ding Y, et al. miR-190 suppresses breast cancer metastasis by regulation of TGF- $\beta$-induced epithelial-mesenchymal transition. Molecular cancer. 2018;17(1):1-12.

88. Lanz TV, Ding Z, Ho PP, Luo J, Agrawal AN, Srinagesh H, et al. Angiotensin II sustains brain inflammation in mice via TGF- $\beta$. The Journal of clinical investigation. 2010;120(8):2782-94.

89. Jiang J, Xia Y, Liang Y, Yang M, Zeng W, Zeng X. miR-190a-5p participates in the regulation of hypoxia-induced pulmonary hypertension by targeting KLF15 and can serve as a biomarker of diagnosis and prognosis in chronic obstructive pulmonary disease complicated with pulmonary hypertension. International Journal of Chronic Obstructive Pulmonary Disease. 2018;13:3777.

90. Jin C, Feng Y, Ni Y, Shan Z. MicroRNA-610 suppresses osteosarcoma oncogenicity via targeting TWIST1 expression. Oncotarget. 2017;8(34):56174.

91. Miao R, Dong X, Gong J, Li J, Pang W, Liu Y, et al. Analysis of significant microRNA associated with chronic thromboembolic pulmonary hypertension. Zhonghua yi xue za zhi. 2018;98(18):1397-402.

92. Rashid F, Awan HM, Shah A, Chen L, Shan G. Induction of miR-3648 upon ER stress and its regulatory role in cell proliferation. International journal of molecular sciences. 2017;18(7):1375.

93. Gu H, Chen L, Xue J, Huang T, Wei X, Liu D, et al. Expression profile of maternal circulating microRNAs as non-invasive biomarkers for prenatal diagnosis of congenital heart defects. Biomedicine \& Pharmacotherapy. 2019;109:823-30.

94. Gao Q, Lei F, Zeng Q, Gao Z, Niu P. Functional passenger-strand miRNAs in exosomes derived from human colon cancer cells and their heterogeneous paracrine effects. International journal of biological sciences. 2020;16(6):1044.

95. Wang F, Wang F, Zhang S, Xu X. MicroRNA-325 inhibits the proliferation and induces the apoptosis of $T$ cell acute lymphoblastic leukemia cells in a BAG2-dependent manner. Experimental and Therapeutic Medicine. 2021;21(6):1-8.

96. Lázár L, Nagy B, Molvarec A, Szarka A, Rigó J. Role of hsa-miR-325 in the etiopathology of preeclampsia. Molecular medicine reports. 2012;6(3):597-600. 
97. Yao Y, Zhou Y, Hua Q. circRNA hsa_circ_0018414 inhibits the progression of LUAD by sponging miR6807-3p and upregulating DKK1. Molecular Therapy-Nucleic Acids. 2021;23:783-96.

98. Wang $Y$, Fan $X, X u F$, Zhang $P$, Wei $Y$, Cheng $S$, et al. Expression of miR-551b and its effect on apoptosis in human gastric carcinoma. International Journal of Clinical and Experimental Pathology. 2018;11(5):2912.

99. Kocyigit I, Taheri S, Sener EF, Eroglu E, Ozturk F, Unal A, et al. Serum micro-rna profiles in patients with autosomal dominant polycystic kidney disease according to hypertension and renal function. BMC nephrology. 2017;18(1):1-10.

\section{Tables}

Tables 1-4 are not available with this version

\section{Figures}

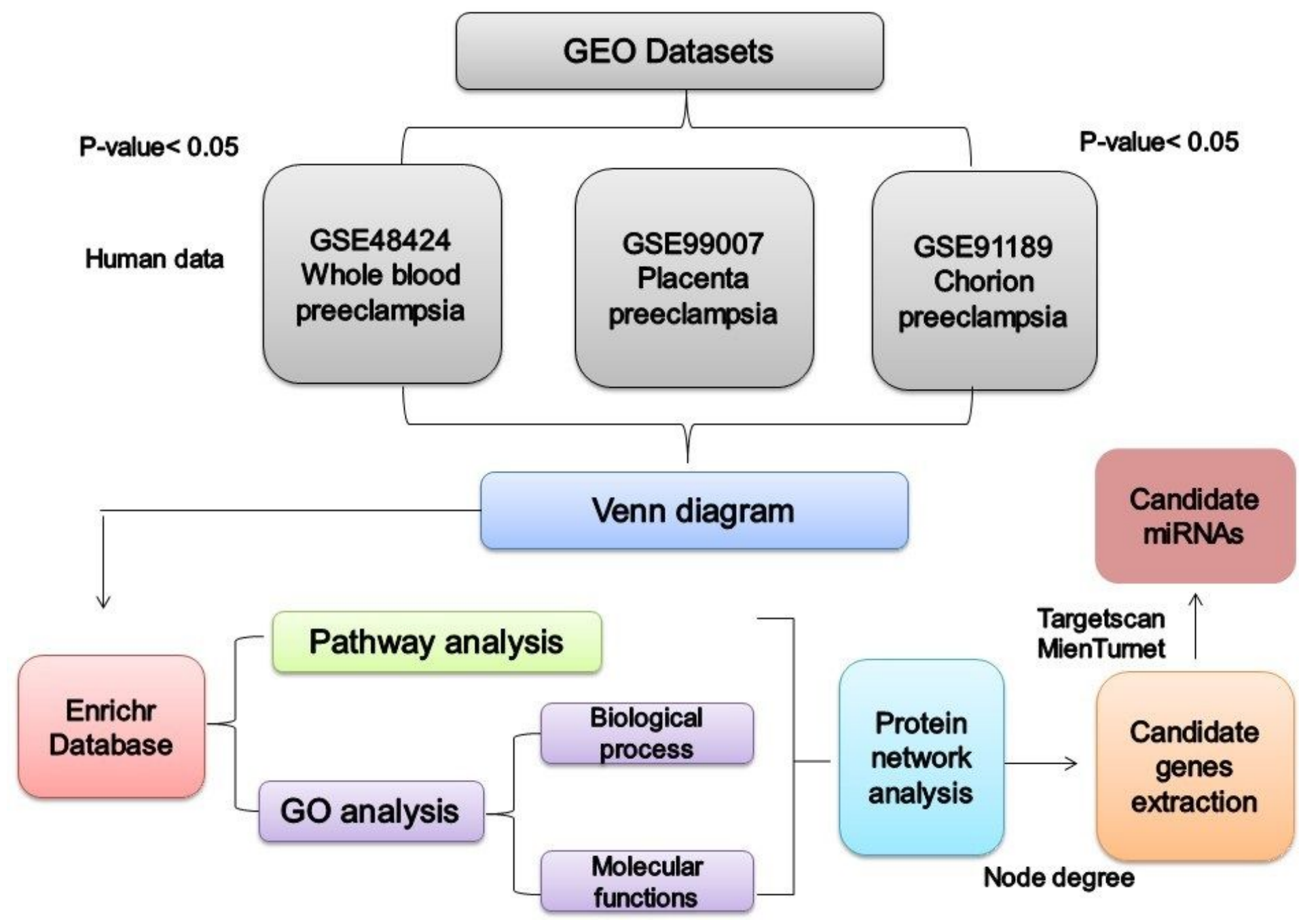

Figure 1 
A

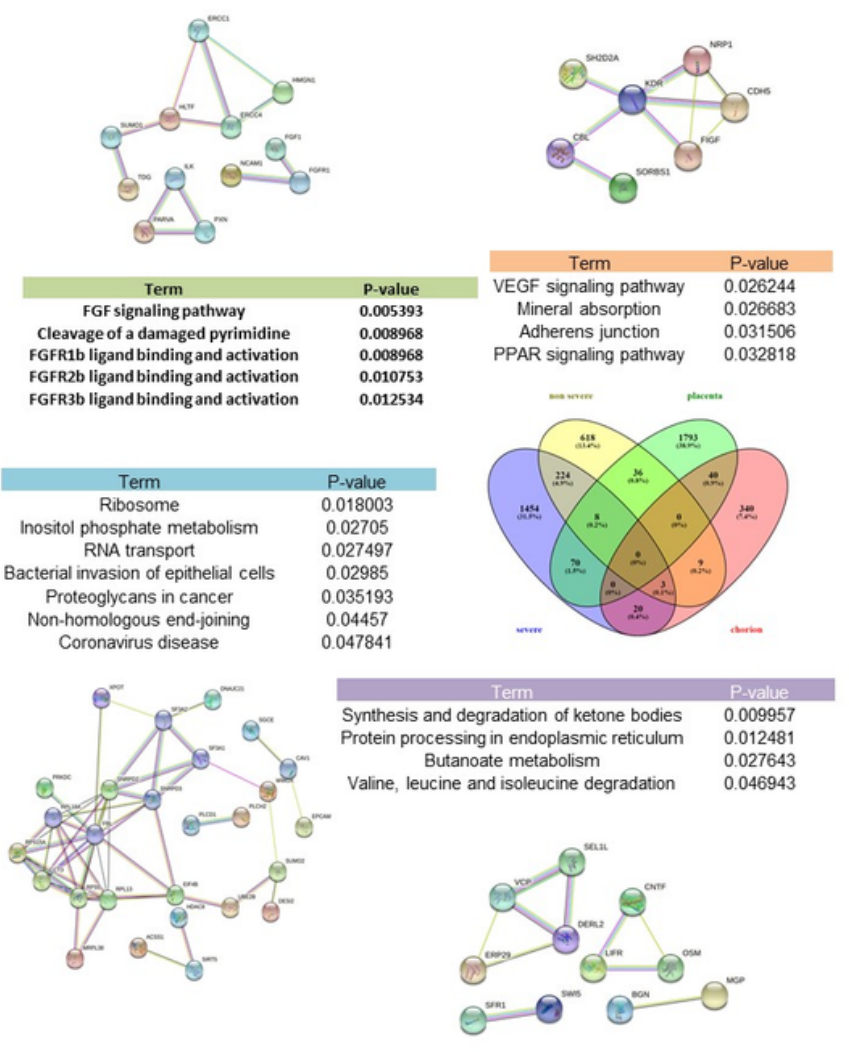

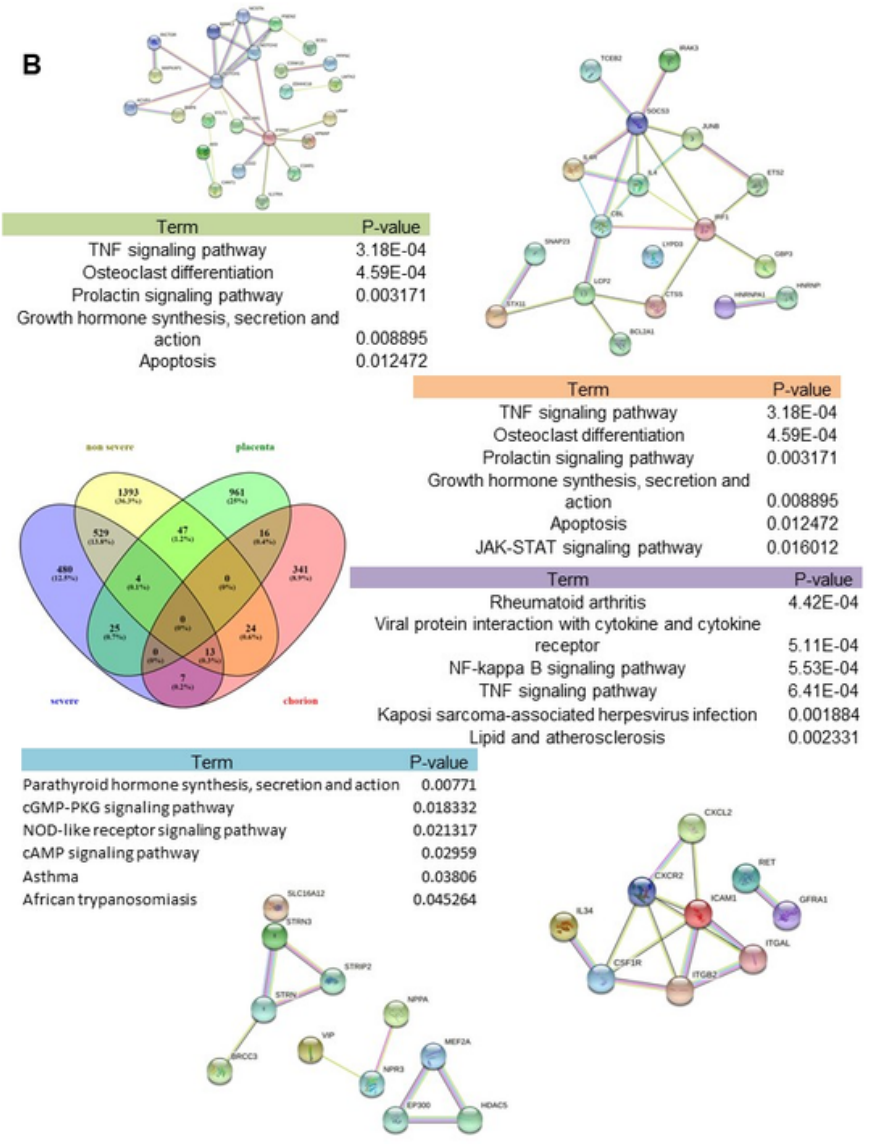

Figure 2

investigation of signaling pathways and proteins network between non-severe to severe preeclampsia with placenta and chorion. A: upregulated genes, B: downregulated genes 
A

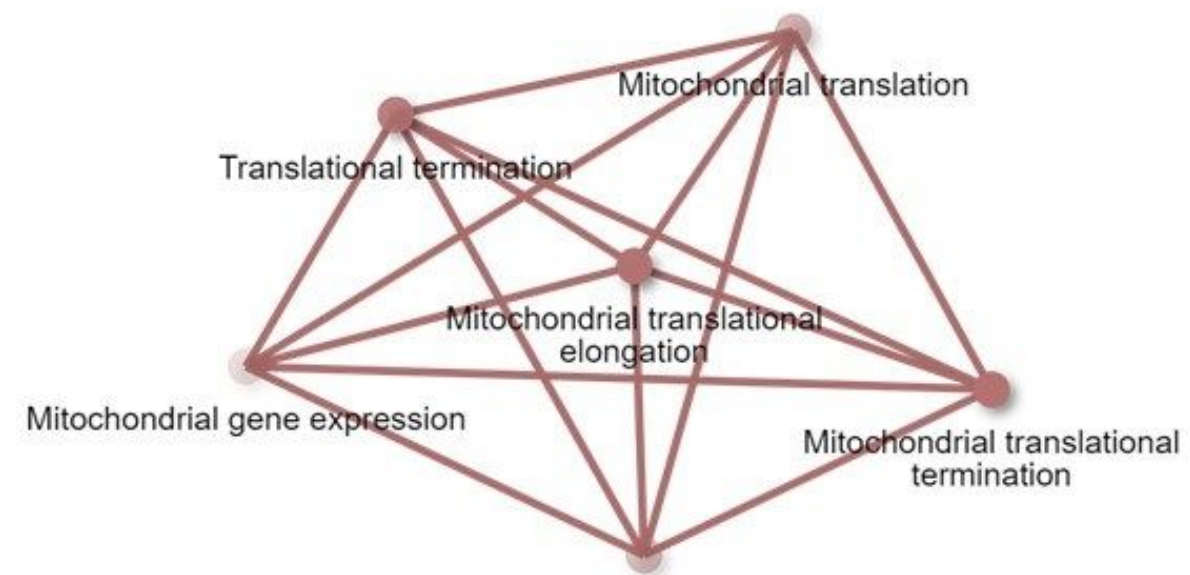

Translational elongation

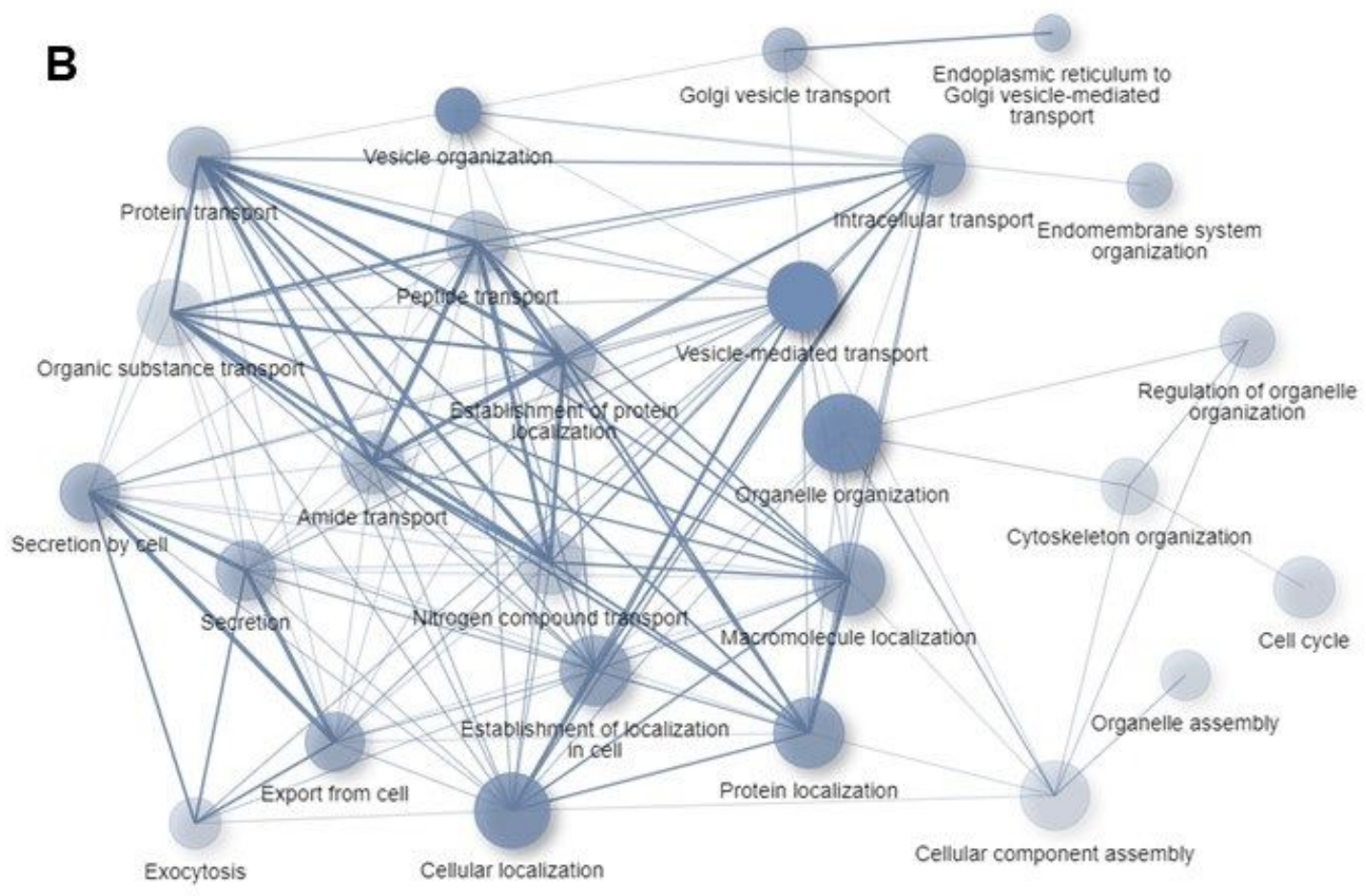

\section{Figure 3}

assessment of biological processes between non-severe to severe preeclampsia. A: upregulated genes, B: downregulated genes 
A

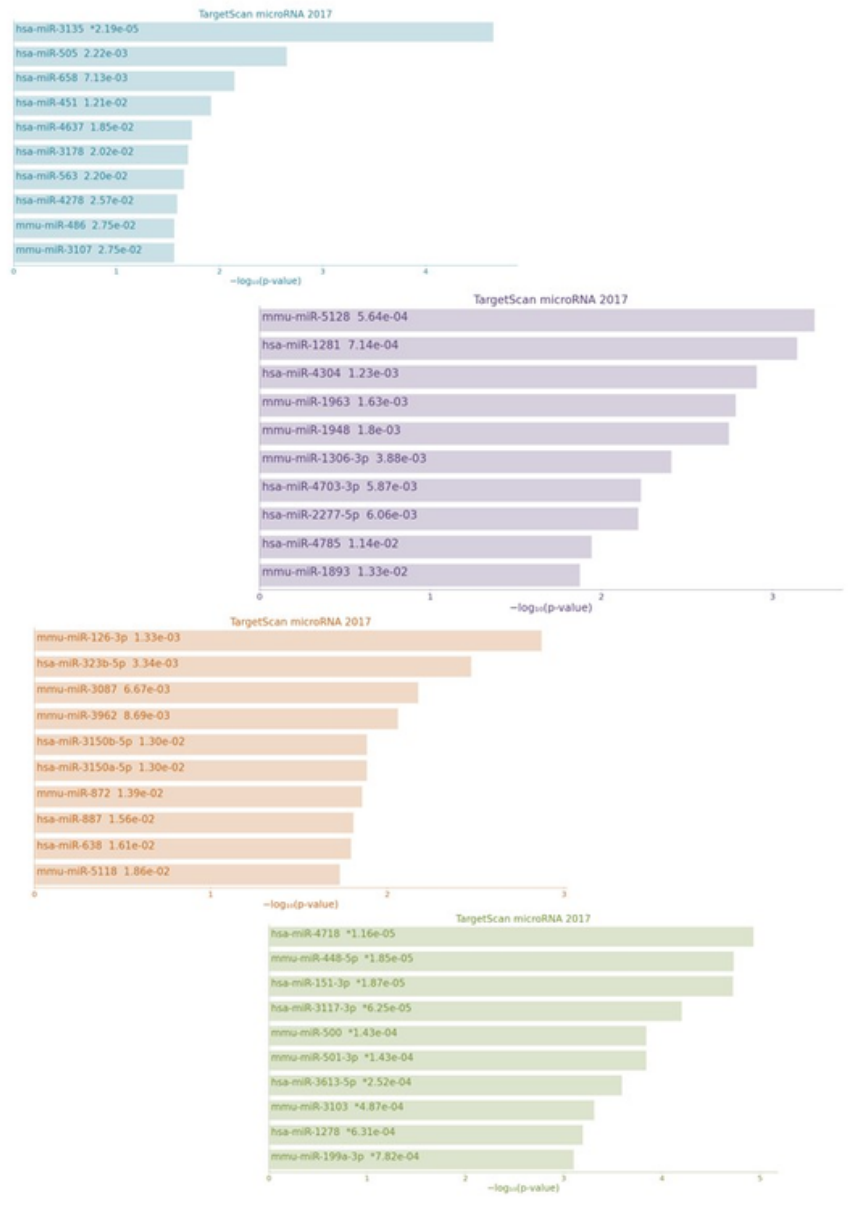

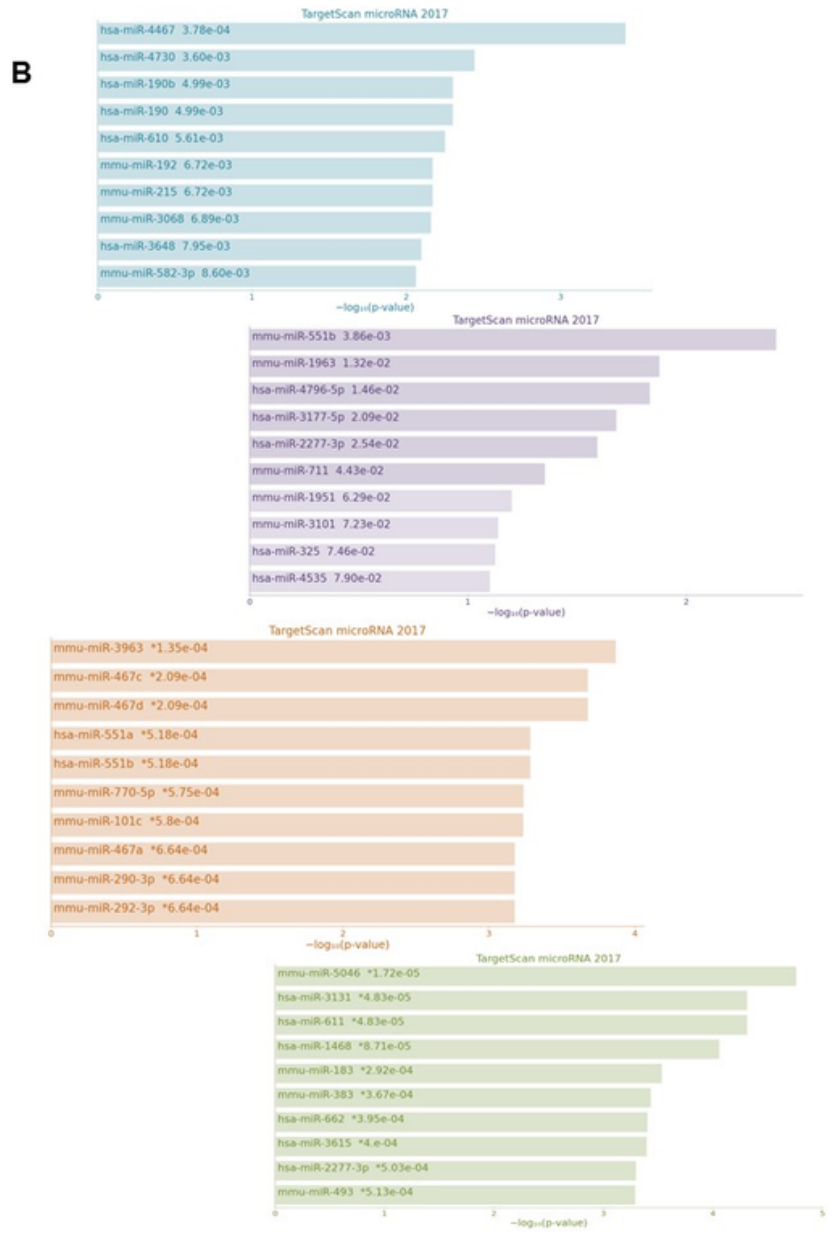

\section{Figure 4}

Analysis of candidate miRNAs between non-severe to severe preeclampsia with placenta and chorion. A: upregulated genes, B: downregulated genes 
A

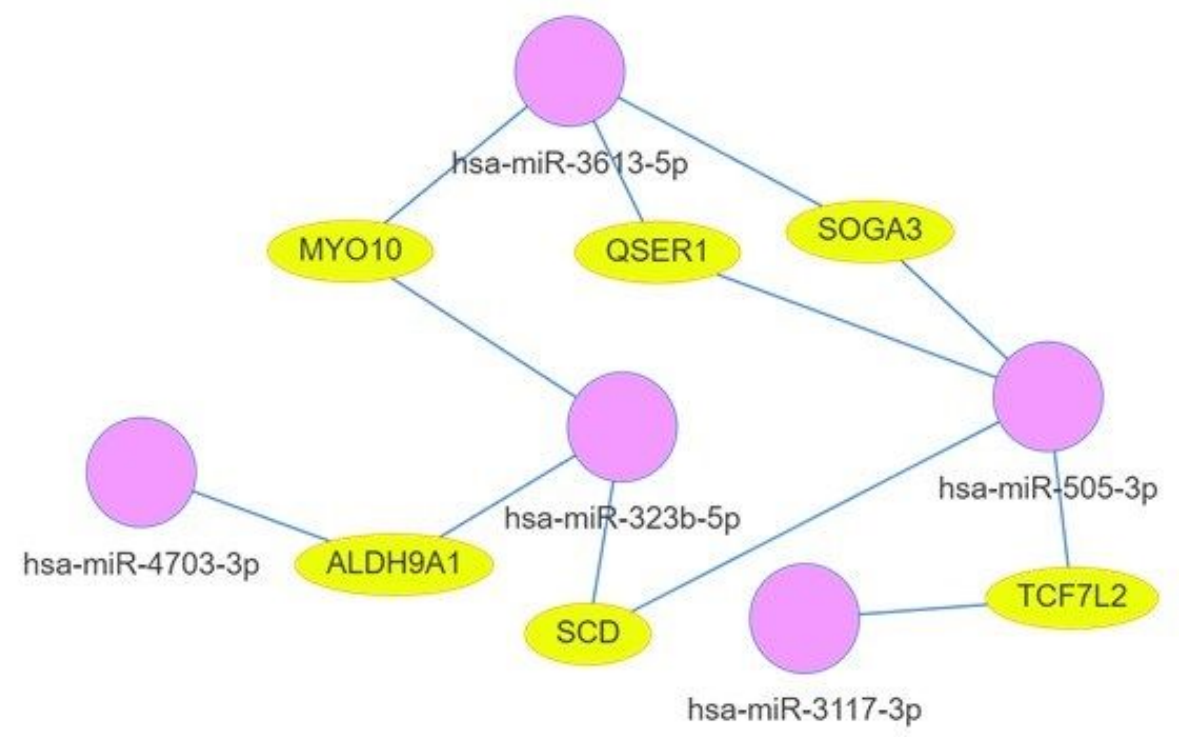

B

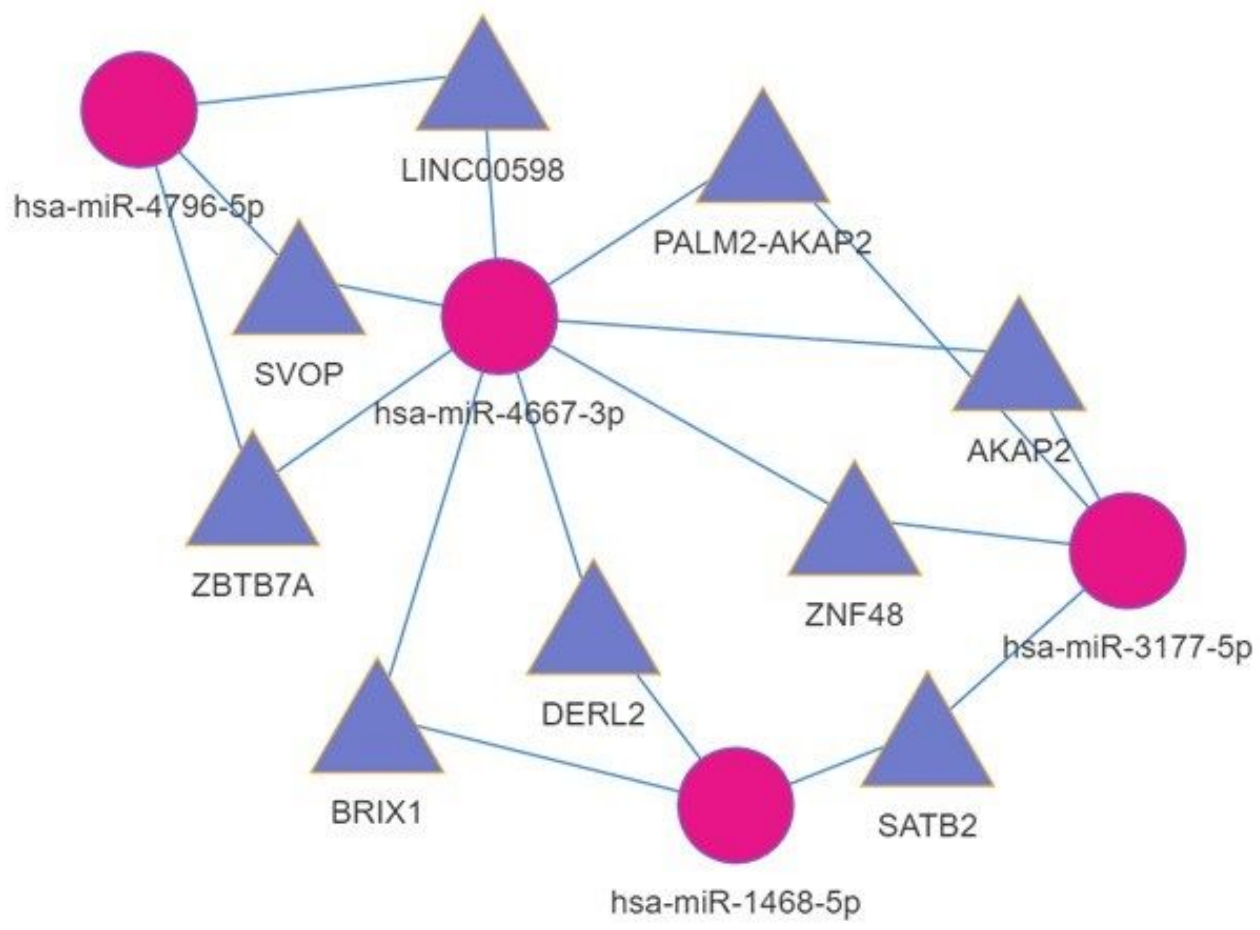

Figure 5

Evaluation of miRNAs networks between hub genes of non-severe to severe preeclampsia with placenta and chorion. A: upregulated genes, B: downregulated genes. 